\title{
Synthesis, Pharmacology, and Structure-Activity Relationships of Novel Imidazolones and Pyrrolones as Modulators of GABA $A_{A}$ Receptors
}

Christian Grunwald, ${ }^{\S}{ }^{*}$ Chris Rundfeldt ${ }^{\S}$ Hans-Joachim Lankau, ${ }^{\S}$ Thomas Arnold, ${ }^{\S}$ Norbert Höfgen, ${ }^{\S}$ Rita Dost, ${ }^{\S}$ Ute Egerland, ${ }^{\S}$ Hans-Jörg Hofmann, ${ }^{\ddagger}$ and Klaus Unverferth ${ }^{\S}$

elbion AG, Meissner Strasse 191, D-01445 Radebeul, Germany, and University of Leipzig, Institute of Biochemistry, Faculty of Biosciences, Pharmacy and Psychology, Brüderstrasse 34, D-04103 Leipzig, Germany

$\S$ elbion AG

* University of Leipzig

* To whom correspondence should be addressed.

Phone: (+49) 351404315 52. Fax: (+49) 35140435552.

E-mail: christian.grunwald@elbion.de

\section{Contents of Supporting Information:}

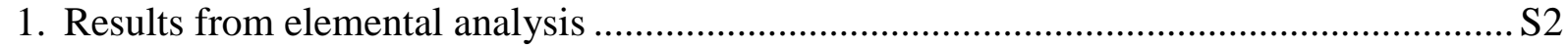

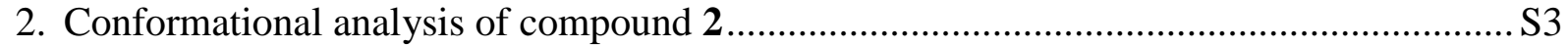

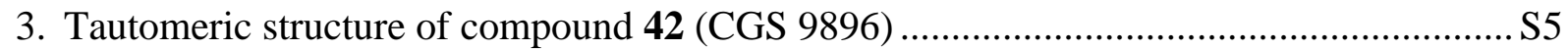

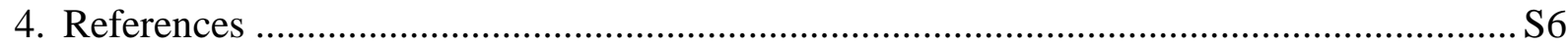




\section{Results from elemental analysis}

Table S1. Elemental analysis data for compounds 1-27, 30-37, 39, 41

\begin{tabular}{|c|c|c|c|c|c|}
\hline Cmpd. & Formula & & $\mathbf{C}$ & $\mathbf{H}$ & $\mathbf{N}$ \\
\hline \multirow[t]{2}{*}{1} & $\mathrm{C}_{13} \mathrm{H}_{15} \mathrm{~N}_{3} \mathrm{O}_{2}$ & Calcd.(\%) & 63.66 & 6.16 & 17.13 \\
\hline & & Found $(\%)$ & 63.53 & 6.21 & 17.07 \\
\hline \multirow[t]{2}{*}{2} & $\mathrm{C}_{13} \mathrm{H}_{14} \mathrm{ClN}_{3} \mathrm{O}_{2}$ & & 55.82 & 5.04 & 15.02 \\
\hline & & & 55.80 & 5.03 & 15.00 \\
\hline \multirow[t]{2}{*}{3} & $\mathrm{C}_{14} \mathrm{H}_{16} \mathrm{ClN}_{3} \mathrm{O}$ & & 60.54 & 5.81 & 15.13 \\
\hline & & & 64.49 & 5.77 & 15.11 \\
\hline \multirow[t]{2}{*}{4} & $\mathrm{C}_{13} \mathrm{H}_{14} \mathrm{ClN}_{3} \mathrm{O}$ & & 59.21 & 5.35 & 15.93 \\
\hline & & & 59.28 & 5.48 & 15.81 \\
\hline \multirow[t]{2}{*}{5} & $\mathrm{C}_{14} \mathrm{H}_{17} \mathrm{~N}_{3} \mathrm{O}_{2}$ & & 64.85 & 6.61 & 16.20 \\
\hline & & & 65.06 & 6.57 & 16.12 \\
\hline \multirow[t]{2}{*}{6} & $\mathrm{C}_{14} \mathrm{H}_{17} \mathrm{ClN}_{4} \mathrm{O}$ & & 57.44 & 5.85 & 19.14 \\
\hline & & & 57.37 & 6.01 & 19.11 \\
\hline \multirow[t]{2}{*}{7} & $\mathrm{C}_{11} \mathrm{H}_{12} \mathrm{ClN}_{3} \mathrm{O}$ & & 55.59 & 5.09 & 17.68 \\
\hline & & & 55.40 & 5.12 & 17.70 \\
\hline \multirow[t]{2}{*}{8} & $\mathrm{C}_{14} \mathrm{H}_{16} \mathrm{ClN}_{3} \mathrm{O}_{2}$ & & 57.24 & 5.49 & 14.30 \\
\hline & & & 57.49 & 5.75 & 14.31 \\
\hline \multirow[t]{2}{*}{9} & $\mathrm{C}_{13} \mathrm{H}_{13} \mathrm{Cl}_{2} \mathrm{~N}_{3} \mathrm{O}_{2}$ & & 49.70 & 4.17 & 13.37 \\
\hline & & & 49.57 & 4.13 & 13.29 \\
\hline \multirow[t]{2}{*}{10} & $\mathrm{C}_{15} \mathrm{H}_{19} \mathrm{~N}_{3} \mathrm{O}_{2}$ & & 65.91 & 7.01 & 15.37 \\
\hline & & & 65.88 & 7.02 & 15.24 \\
\hline \multirow[t]{2}{*}{11} & $\mathrm{C}_{14} \mathrm{H}_{15} \mathrm{Cl}_{2} \mathrm{~N}_{3} \mathrm{O}_{2}$ & & 51.24 & 4.61 & 12.80 \\
\hline & & & 51.23 & 4.80 & 12.84 \\
\hline \multirow[t]{2}{*}{12} & $\mathrm{C}_{13} \mathrm{H}_{14} \mathrm{BrN}_{3} \mathrm{O}_{2}$ & & 48.17 & 4.35 & 12.96 \\
\hline & & & 48.30 & 4.45 & 12.88 \\
\hline \multirow[t]{2}{*}{13} & $\mathrm{C}_{13} \mathrm{H}_{14} \mathrm{IN}_{3} \mathrm{O}_{2}$ & & 42.07 & 3.80 & 11.32 \\
\hline & & & 42.25 & 3.82 & 11.19 \\
\hline \multirow[t]{2}{*}{14} & $\mathrm{C}_{13} \mathrm{H}_{14} \mathrm{FN}_{3} \mathrm{O}_{2}$ & & 59.31 & 5.36 & 15.96 \\
\hline & & & 59.25 & 5.33 & 15.90 \\
\hline \multirow[t]{2}{*}{15} & $\mathrm{C}_{13} \mathrm{H}_{14} \mathrm{ClN}_{3} \mathrm{O}_{2}$ & & 55.82 & 5.04 & 15.02 \\
\hline & & & 55.94 & 5.05 & 15.08 \\
\hline \multirow[t]{2}{*}{16} & $\mathrm{C}_{15} \mathrm{H}_{18} \mathrm{ClN}_{3} \mathrm{O}$ & & 61.75 & 6.22 & 14.40 \\
\hline & & & 61.78 & 6.15 & 14.60 \\
\hline \multirow[t]{2}{*}{17} & $\mathrm{C}_{16} \mathrm{H}_{20} \mathrm{ClN}_{3} \mathrm{O}$ & & 62.84 & 6.59 & 13.74 \\
\hline & & & 62.69 & 6.67 & 13.99 \\
\hline \multirow[t]{2}{*}{18} & $\mathrm{C}_{15} \mathrm{H}_{19} \mathrm{~N}_{3} \mathrm{O}_{2}$ & & 65.91 & 7.01 & 15.37 \\
\hline & & & 65.98 & 7.16 & 15.45 \\
\hline \multirow[t]{2}{*}{19} & $\mathrm{C}_{16} \mathrm{H}_{20} \mathrm{ClN}_{3} \mathrm{O}$ & & 62.84 & 6.59 & 13.74 \\
\hline & & & 62.71 & 6.52 & 13.75 \\
\hline \multirow[t]{2}{*}{20} & $\mathrm{C}_{14} \mathrm{H}_{15} \mathrm{Cl} \mathrm{N} \mathrm{N}_{2} \mathrm{O}_{2}$ & & 60.33 & 5.42 & 10.05 \\
\hline & & & 60.21 & 5.62 & 9.88 \\
\hline \multirow[t]{2}{*}{21} & $\mathrm{C}_{14} \mathrm{H}_{15} \mathrm{Cl} \mathrm{N} \mathrm{N}_{2}$ & & 64.00 & 5.75 & 10.66 \\
\hline & & & 63.74 & 5.69 & 10.64 \\
\hline \multirow[t]{2}{*}{22} & $\mathrm{C}_{16} \mathrm{H}_{19} \mathrm{IN}_{2} \mathrm{O}$ & & 66.09 & 6.59 & 9.63 \\
\hline & & & 66.01 & 6.60 & 9.65 \\
\hline
\end{tabular}




\begin{tabular}{|c|c|c|c|c|c|}
\hline Cmpd. & Formula & & $\mathbf{C}$ & $\mathbf{H}$ & $\mathbf{N}$ \\
\hline \multirow[t]{2}{*}{23} & $\mathrm{C}_{15} \mathrm{H}_{17} \mathrm{ClN}_{2} \mathrm{O}$ & Calcd. $(\%)$ & 65.10 & 6.19 & 10.12 \\
\hline & & Found (\%) & 65.02 & 6.12 & 10.11 \\
\hline \multirow[t]{2}{*}{24} & $\mathrm{C}_{14} \mathrm{H}_{15} \mathrm{FN}_{2} \mathrm{O}_{2}$ & & 64.11 & 5.76 & 10.68 \\
\hline & & & 64.05 & 5.82 & 10.66 \\
\hline \multirow[t]{2}{*}{25} & $\mathrm{C}_{15} \mathrm{H}_{18} \mathrm{~N}_{2} \mathrm{O}_{2}$ & & 69.74 & 7.02 & 10.84 \\
\hline & & & 69.76 & 7.03 & 10.82 \\
\hline \multirow[t]{2}{*}{26} & $\mathrm{C}_{15} \mathrm{H}_{18} \mathrm{~N}_{2} \mathrm{O}_{2}$ & & 69.74 & 7.02 & 10.84 \\
\hline & & & 69.65 & 7.00 & 10.88 \\
\hline \multirow[t]{2}{*}{27} & $\mathrm{C}_{14} \mathrm{H}_{14} \mathrm{ClFN}_{2} \mathrm{O}_{2}$ & & 56.67 & 4.76 & 9.44 \\
\hline & & & 56.55 & 4.68 & 9.40 \\
\hline \multirow[t]{2}{*}{30} & $\mathrm{C}_{15} \mathrm{H}_{15} \mathrm{ClN}_{2} \mathrm{O}_{2}$ & & 61.97 & 5.20 & 9.63 \\
\hline & & & 61.47 & 5.22 & 9.64 \\
\hline \multirow[t]{2}{*}{31} & $\mathrm{C}_{14} \mathrm{H}_{13} \mathrm{ClN}_{2} \mathrm{O}_{2}$ & & 60.77 & 4.74 & 10.12 \\
\hline & & & 60.55 & 4.64 & 10.14 \\
\hline \multirow[t]{2}{*}{32} & $\mathrm{C}_{14} \mathrm{H}_{13} \mathrm{FN}_{2} \mathrm{O}_{3}$ & & 60.87 & 4.74 & 10.14 \\
\hline & & & 60.98 & 5.26 & 10.21 \\
\hline \multirow[t]{2}{*}{33} & $\mathrm{C}_{15} \mathrm{H}_{15} \mathrm{FN}_{2} \mathrm{O}_{2}$ & & 65.67 & 5.52 & 10.22 \\
\hline & & & 65.23 & 5.96 & 10.15 \\
\hline \multirow[t]{2}{*}{34} & $\mathrm{C}_{14} \mathrm{H}_{13} \mathrm{FN}_{2} \mathrm{O}_{2}$ & & 64.61 & 5.03 & 10.76 \\
\hline & & & 64.34 & 5.35 & 10.54 \\
\hline \multirow[t]{2}{*}{35} & $\mathrm{C}_{14} \mathrm{H}_{10} \mathrm{ClN}_{3}$ & & 65.87 & 3.95 & 16.47 \\
\hline & & & 65.74 & 4.02 & 16.39 \\
\hline \multirow[t]{2}{*}{36} & $\mathrm{C}_{12} \mathrm{H}_{12} \mathrm{ClN}_{3} \mathrm{O}_{2}$ & & 54.33 & 4.56 & 15.85 \\
\hline & & & 54.28 & 4.52 & 15.81 \\
\hline \multirow[t]{2}{*}{37} & $\mathrm{C}_{15} \mathrm{H}_{10} \mathrm{ClNO}_{2}$ & & 66.31 & 3.71 & 5.16 \\
\hline & & & 66.22 & 3.72 & 5.17 \\
\hline \multirow[t]{2}{*}{39} & $\mathrm{C}_{15} \mathrm{H}_{18} \mathrm{ClN}_{3} \mathrm{O}_{4}$ & & 53.08 & 5.35 & 12.39 \\
\hline & & & 52.89 & 5.42 & 12.11 \\
\hline \multirow[t]{2}{*}{41} & $\mathrm{C}_{14} \mathrm{H}_{9} \mathrm{ClN}_{2} \mathrm{O}_{2}$ & & 61.76 & 3.33 & 10.30 \\
\hline & & & 61.63 & 3.41 & 10.19 \\
\hline
\end{tabular}

\section{Conformational analysis of compound 2}

The planar structure of compound 2 was confirmed by an extensive conformational analysis at various semi-empirical and ab initio MO approximation levels. Semi-empirical AM1 and PM3 calculations were done by means of the program VAMP ${ }^{1}$ using an increment of $5^{\circ}$ for the torsion angles (Figure S1). Geometry optimizations at the $\mathrm{HF} / 3-21 \mathrm{G}^{(*)}$ (contour maps) and HF/6-31G* basis set levels of ab initio MO theory were performed employing the programs SPARTAN ${ }^{2}$ and GAUSSIAN ${ }^{3}$, respectively, with torsion angle increments of $30^{\circ}$, using the AM1 minimum structures as starting points. A good correlation was found between the ab initio and the AM1 results, but these were not so well correlated with the PM3 data. Therefore, AM1 was used throughout this study. 


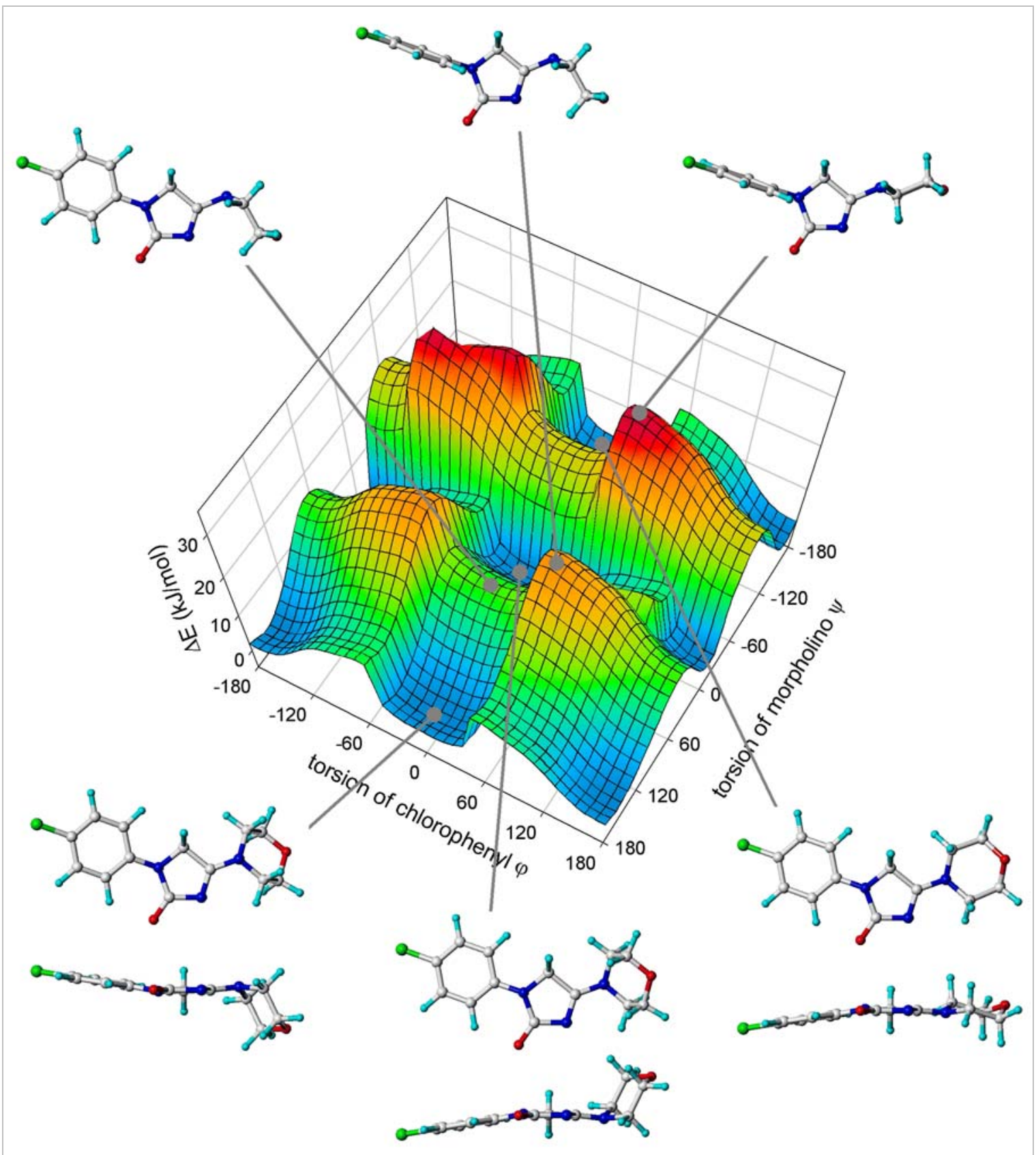

Figure S1. Relative heat of formation $\Delta \mathrm{E}$ as a function of torsion angles from conformational analysis of 2 using AM1. Some geometries corresponding to stationary points (maxima, minima, saddle points) on the surface are depicted. 


\section{Tautomeric structure of compound 42 (CGS 9896)}

42 was used as reference structure and template for the alignment. Therefore, the structure had to be studied in detail. Two keto tautomers $\mathbf{T 1}$ and $\mathbf{T 2}$ and two enolic structures T3 and T3a may exist as can be seen in Figure S2. To find the energetically favored state, calculations were done using semi-empirical AM1 and PM3 methods and $a b$ initio methods at the $\mathrm{HF} / 3-21 \mathrm{G}^{(*)}$ and $\mathrm{HF} / 6-31 \mathrm{G}^{*}$ levels. The solvent influence was estimated at the SCI/6-31G* and SCRF/6-31G* levels for the solvent water. The results are listed in Table S2. It can be seen that the two enolic tautomers are very unfavorable. All methods except PM3 gave the result that the keto tautomer T2 is energetically favored over T1. This confirms the priority of AM1 over PM3 regarding the compounds of this study.<smiles>[R]c1ccc(-n2[nH]c3c(cnc4ccccc43)c2=O)cc1</smiles>

T1<smiles>[R]c1ccc(-n2nc3c4ccccc4[nH]cc-3c2=O)cc1</smiles>

T2<smiles>[R]c1ccc(-n2nc3c(cnc4ccccc43)c2O)cc1</smiles>

T3<smiles>[R]c1ccc(-n2nc3c(cnc4ccccc43)c2O)cc1</smiles>

T3a

Figure S2. Tautomers of $\mathbf{4 2}$

Table S2. Relative Stabilities ${ }^{\mathrm{a}}$ of $\mathbf{4 2}$ Tautomers at Various Levels of MO Theory

\begin{tabular}{|c|c|c|c|c|}
\hline Method & T1 & $\mathbf{T} 2$ & T3 & T3a \\
\hline AM1 & 9.9 & $0.0^{\mathrm{b}}$ & 33.5 & 33.3 \\
\hline PM3 & $0.0^{c}$ & 16.0 & 38.8 & 27.2 \\
\hline $\mathrm{HF} / 3-21 \mathrm{G}^{(*)}$ & 27.5 & $\mathbf{0 . 0 ^ { \mathrm { d } }}$ & $-^{\mathrm{e}}$ & 74.4 \\
\hline $\mathrm{HF} / 6-31 \mathrm{G}^{*}$ & 2.1 & $0.0^{\mathrm{f}}$ & 48.4 & 47.4 \\
\hline $\mathrm{SCI} / 6-31 \mathrm{G}^{* \mathrm{~g}}$ & 11.8 & $0.0^{h}$ & $-{ }^{\mathrm{i}}$ & 55.2 \\
\hline SCRF/6-31G ${ }^{* j}$ & 58.9 & $0.0^{\mathrm{k}}$ & 114.4 & 118.4 \\
\hline
\end{tabular}

${ }^{\mathrm{a}}$ In kJ/mol. ${ }^{\mathrm{b}} \Delta H=417.7 \mathrm{~kJ} / \mathrm{mol}^{\mathrm{c}}{ }^{\mathrm{c}} \Delta H=265.7 \mathrm{~kJ} / \mathrm{mol} .{ }^{\mathrm{d}} E_{\mathrm{T}}=-1302.582452$ a. u. ${ }^{\mathrm{e}}$ The calculation yielded T3a. ${ }^{\mathrm{f}} E_{\mathrm{T}}=-1309.409534$ a. u. ${ }^{\mathrm{g}} \mathrm{A}_{0}=0.001, \varepsilon=78.4$, SCI/HF/6-31G*. ${ }^{\mathrm{h}} E_{\mathrm{T}}=-1309.438047$ a. u. ${ }^{\mathrm{i}}$ Not calculated. ${ }^{\mathrm{j}}$ Molecular radius $r=4.57$ $\AA$ A, dielectric constant $\varepsilon=78.4 .{ }^{\mathrm{k}} E_{\mathrm{T}}=-1309.437421$ a. u. 


\section{References}

(1) Rauhut, G., Alex, A., Chandrasekhar, J., Steinke, T., Sauer, W., Beck, B., Hutter, M., Gedeck, M., and Clark, T. VAMP version 6.5, 1997. The Medawar Centre, Oxford Science Park, Oxford OX4 4GA, UK, Oxford Molecular Group.

(2) SPARTAN version 4.1 and 5.1, 1999. 18401 Von Karman Avenue, Suite 370, Irvine, CA 92612, USA, Wavefunction, Inc.

(3) Frisch, M. J., Trucks, G. W., Schlegel, H. B., Gill, P. M. W., Johnson, B. G., Robb, M. A., Cheeseman, J. R., Keith, T. A., Petersson, G. A., Montgomery, J. A., Raghavachari, K., Al-Laham, M. A., Zakrzewski, V. G., Ortiz, J. V., Foresman, J. B., Peng, C. Y., Ayala, P. Y., Wong, M. W., Andres, J. L., Replogle, E. S., Gomperts, R., Martin, R. L., Fox, D. J., Binkley, J. S., Defrees, D. J., Baker, J., Stewart, J. P., Head-Gordon, M., Gonzalez, C., and Pople, J. A. GAUSSIAN94, 1995. Pittsburgh PA, USA, Gaussian, Inc. 